\title{
Positive acceleration adaptive training attenuates gastric ischemia-reperfusion injury through COX-2 and PGE2 expression
}

\author{
YING CHEN, JIAN-CHANG WANG, CHUN-MIN YANG, QIN FAN, JUN ZHENG and HAO LIU \\ Department of Gastroenterology and Research Center of Aeropathy, \\ General Hospital of The Air Force, People's Liberation Army, Beijing 100036, P.R. China
}

Received May 3,2017; Accepted March 2, 2018

DOI: $10.3892 /$ etm.2019.7288

\begin{abstract}
The mechanism involved in the effects of positive acceleration adaptive training (PAAT) on gastric ischemia-reperfusion injury (GI-RI) has not been fully characterized. The aim of the present study was to investigate the effects of PAAT in attenuating GI-RI in a rat model. The inflammatory factor and caspase-3 levels were measured using ELISA kits. A western blot assay was used to analyze tumor necrosis factor- $\alpha$ (TNF)- $\alpha$, tumor necrosis factor receptor 1 (TNFR1), tumor necrosis factor-related apoptosis inducing ligand (TRAIL), death receptor (DR) 4, DR5, cyclooxygenase (COX)-2, COX-1 and prostaglandin E2 (PGE2) protein expression levels. It was revealed that PAAT could alleviate GI-RI and inflammatory factor levels in a rat model. PAAT suppressed TNF- $\alpha$ and TNFR1 protein expression levels, inhibited TRAIL, DR4, DR5, COX-2 and PGE2 protein expression levels; however, it did not have an effect on COX-1 protein expression in the model of GI-RI. The data indicated that the effects of PAAT attenuated GI-RI through the downregulation of COX-2 and PGE2 expression.
\end{abstract}

\section{Introduction}

Gastric ischemia-reperfusion injury (GI-RI) is caused by the restoration of a blood supply following short-term stomach blood supply deficiency (1). It is commonly observed in critically ill patients (1). GI-RI is closely associated with the mechanism of stress-related mucosal disease (SRMD) due to severe trauma, burns, shock, major surgery and critical illness (1). Additionally, SRMD may further result in prolonged hospitalization, mental pain and heavy economic burden. It may also significantly

Correspondence to: Dr Chun-Min Yang, Department of Gastroenterology and Research Center of Aeropathy, General Hospital of The Air Force, People's Liberation Army, 30 Fucheng Road, Beijing 100036, P.R. China

E-mail: yylnwwm86387@126.com

Key words: positive acceleration adaptive training, gastric ischemia-reperfusion injury, cyclooxygenase-2, prostaglandin E2 increase mortality if complicated with stress ulcer bleeding and enterogenous infection, which has led to the consumption of lots of medical resources (2). Preventing and relieving SRMD and maintaining the whole physiological status has been the focus of emergency and critical care medicine and nursing (3).

Prostaglandin (PG) E2 is a PG with complex biological activity, which is involved in GI-RI inflammation (4). PGE2 synthase is the last key enzyme in the synthesis of PGE2. PGE2 is the PG with the highest abundance and greatest distribution in the human body (5). It serves a major role in inflammation as a pain and fever mediator during inflammation. Additionally, it may induce vasodilation and microvascular leakage (5). As a type of unsaturated fatty acid, PGE2 is predominantly composed of 20 carbon atoms (6). It has the basic structure of one five-carbon ring and two side chains. Arachidonic acid is synthesized into PGE2 under the catalysis of cyclooxygenase (COX) and PG synthase (6). PGE2 escapes through facilitated diffusion and binds to E-prostanoid 1-4 in an autocrine or paracrine manner. In this way, it may alter the levels of intracellular second messengers, and send signals to cells, causing a series of physiological or pathophysiological changes (4).

Cyclooxygenase prevents the conversion of arachidonic acid into PGE2 (7). Therefore, it may quickly alleviate active substance-induced inflammation. As a result, it may reduce the excitability of the peripheral and central pain sensing conduction system (7). Thus, COX-2 serves anti-inflammatory and analgesic functions. PGs may promote the secretion of gastric fluid and bicarbonate. In this manner, they are able to protect the gastric mucosal barrier, promote the renewal of gastric mucosal cells and improve mucosal blood flow (8). Furthermore, COX-2 may stimulate the active transport process of cells, activate adenylate cyclase and stabilize the lysosome (8). It may also maintain the level of mucosal thiol compounds and stimulate surface-active phospholipids in gastric mucosa (8). Previous study has revealed that the role of $\mathrm{COX}$ in the protection of gastric mucosa is not only associated with the PGs that it produces, but is more related to a variety of growth factors that it secretes, which act on fibroblasts (8). Additionally, COX-2 may stimulate the synthesis and secretion of hepatocyte growth factors and vascular endothelial growth factors, and promote the repair of tissue injury $(8,9)$.

Positive acceleration adaptive training (PAAT) is a training method utilized by fighter pilots. Improving the load 
resistance protective capability of high-performance fighter pilots is a primary focus of aviation medicine research (10). In the field of aerospace medicine, the alleviation of PAAT damage to pilot systemic organs has been a topic of extensive research (11). Previous studies have demonstrated that pre-adaptive training mitigates damage to gastric mucosa of PAAT exposed rats $(10,11)$. However, the precise mechanism of action is yet to be elucidated. Therefore, the aim of the present study was to determine the effects of positive acceleration adaptive training (PAAT) on GI-RI in a rat model.

\section{Materials and methods}

Models of ischemia/reperfusion(I/R)-induced gastric injury. A total of 24 Male Sprague-Dawley rats (200-230 g, 8-10 weeks) were supplied by the Experimental Animal Center at Peking University (Beijing, China). Rats were housed in $22-24^{\circ} \mathrm{C}$ at a relative humidity of $60 \pm 10 \%$ under 12 -h light/dark cycles. Rats were sustained on standard rat chow and tap water ad libitum. All experimental procedures were performed under the Care of Laboratory Animals and approved by the Animal Care and Use Committee of People's Liberation Army (Beijing, China) (12). The rats were randomly divided into three groups ( $\mathrm{n}=8$ /group): Sham operation, GI-RI model (gastric model) and PAAT (gastric I/R injury) groups. In the PAAT group, the rats were trained using PAAT for 7 days. Firstly, the rat was fixed with a set-up box at a centrifuge with a radius of $1 \mathrm{~m}$ and the head of the rat was orientated towards the axis of the centrifuge at $5 \mathrm{x}$ g/day for 7 days (13). Subsequently, gastric I/R injury was performed. The rats were injected with $400 \mathrm{mg} / \mathrm{kg}$ chloral hydrate (intraperitoneal; Sigma-Aldrich; Merck KGaA, Darmstadt, Germany) and the abdominal cavity was opened. Celiac arterial trunk tissues were cautiously isolated and clamped with a small vascular clamp for $1 \mathrm{~h}$. This procedure was also performed in the GI-RI model group, without prior PAAT. In the sham operation group, the rats were injected with $400 \mathrm{mg} / \mathrm{kg}$ chloral hydrate and the abdominal cavity was only opened.

Histopathological measurements. The rats were sacrificed and the stomach was removed, washed with PBS three times and fixed with $4 \%$ paraformaldehyde for $24 \mathrm{~h}$ at room temperature. Tissue samples were embedded in paraffin, sliced to $5-\mathrm{mm}$ thickness and stained with hematoxylin and eosin for $5 \mathrm{~min}$ at room temperature. All samples were imaged using a laser scanning confocal microscope (magnification, $\mathrm{x} 20$; TCS SP5; Leica Microsystems GmbH, Wetzlar, Germany).

Measurement of inflammation and caspase-3 levels using ELISA kits. Blood was collected and centrifuged at 3,000 x g for $10 \mathrm{~min}$ at $4^{\circ} \mathrm{C}$. Serum was collected and used to determine the levels of interleukin (IL)-1 $\beta$ (cat. no. H002), IL-6 (cat. no. H007), tumor necrosis factor- $\alpha$ (TNF- $\alpha$; cat. no. H052) and nuclear factor- $\kappa \mathrm{B}(\mathrm{NF}-\kappa \mathrm{B}, \mathrm{H} 202)$ using ELISA kits (Nanjing Jiancheng Biology Engineering Institute, Nanjing, China). Total protein was extracted from gastric tissues using radioimmunoprecipitation assay (RIPA; Beyotime Institute of Biotechnology, Haimen, China) buffer and quantified using a bicinchoninic acid (BCA) assay. A total of $10 \mu \mathrm{g}$ protein was used to measure the caspase-3 levels using an ELISA kit.
Western blot assay. Total protein was extracted from gastric tissues using lysis buffer (RIPA) and quantified using a BCA assay. Proteins $(50 \mu \mathrm{g})$ were separated by $8-12 \%$ SDS-PAGE, transferred onto polyvinylidene difluoride membranes (EMD Millipore, Billerica, MA, USA), and blocked with 5\% dried skimmed-milk in Tris-buffered saline and $0.1 \%$ Tween 20 (Sangon Biotech Co., Ltd., Shanghai, China) for $1 \mathrm{~h}$ at $37^{\circ} \mathrm{C}$. Subsequently, membranes were incubated overnight at $4^{\circ} \mathrm{C}$ with TNF- $\alpha$ (1:500; sc-8301), tumor necrosis factor receptor 1 (TNFR1; 1:500; sc-7895), tumor necrosis factor-related apoptosis inducing ligand (TRAIL; 1:500; sc-7877), death receptor (DR)4 (1:500; sc-7863), DR5 (1:500; sc-166624), COX-2 (1:500; sc-7951), COX-1 (1:500; sc-7950), PGE2 (1:500; sc-20676) and GAPDH (1:500; sc-25778), all purchased from Santa Cruz Biotechnology, Inc. (Dallas, TX, USA). Following this, the membranes were individually incubated for $2 \mathrm{~h}$ at room temperature with Horseradish peroxidase conjugated goat anti-rabbit Immunoglobulin G (1:5,000, cat. no. sc-2004; Santa Cruz Biotechnology, Inc.). Protein bands were visualized using an enhanced chemiluminescence system (Beyotime Institute of Biotechnology) and imaged using Quantity One software version 4.62 (Bio-Rad Laboratories, Inc., Hercules, CA, USA).

Statistical analysis. All data were expressed as the mean \pm standard deviation using SPSS 21.0 (IBM Corp., Armonk, NY, USA). Statistical analysis of the quantitative data for multiple group comparisons was performed using one-way analysis of variance followed by Duncan's test. $\mathrm{P}<0.05$ was considered to indicate a statistically significant difference.

\section{Results}

PAAT alleviates GI-RI. Compared with the sham group, stomach cells appeared damaged in GI-RI model group. In addition, GI-RI was successfully established in the model and it was demonstrated that PAAT alleviated this injury (Fig. 1). PAAT also effectively alleviated GI-RI compared with the GI-RI model (Fig. 1). These results demonstrated that PAAT prevented GI-RI; however, its mechanism is yet to be elucidated.

PAAT reduces inflammatory factors in rats with GI-RI. The levels of inflammatory factors were measured and the results demonstrated that there were significant increases of IL-1 $\beta$, IL-6, TNF- $\alpha$ and NF- $\kappa$ B serum levels in the GI-RI model compared with the levels in the sham group (Fig. 2). PAAT significantly alleviated IL- $1 \beta$, IL- $6, \mathrm{TNF}-\alpha$ and NF- $\kappa \mathrm{B}$ serum levels in rats with GI-RI compared with the levels in the GI-RI model (Fig. 2). Therefore, these results demonstrated that PAAT reduced inflammation in GI-RI.

PAAT suppresses caspase-3 activity in rats with GI-RI. As demonstrated in Fig. 3, the caspase-3 activity in rats with GI-RI was significantly increased compared with the level in the sham group. However, PAAT significantly suppressed the caspase-3 activity in rats with GI-RI compared with that in the GI-RI model group (Fig. 3). These results indicate that PAAT may reduce GI-RI-induced apoptosis in a rat model. 


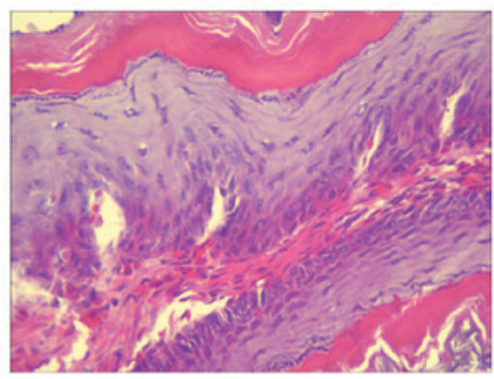

Sham

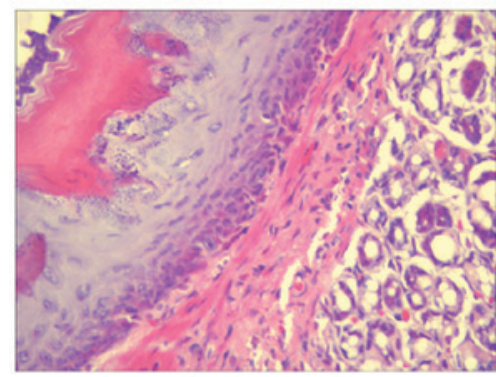

Gastric model

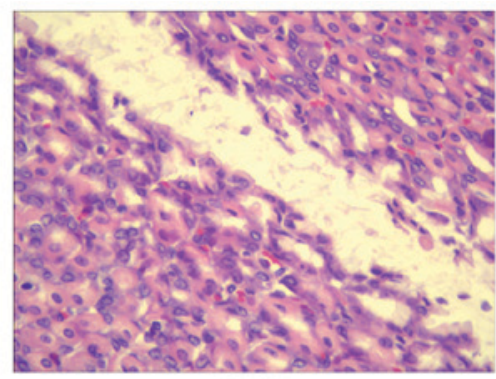

PAAT

Figure 1. PAAT alleviates gastric ischemia-reperfusion injury and recovers gastric epithelial cells. Sham, sham control group; Gastric, gastric ischemia-reperfusion injury group; PAAT, positive acceleration adaptive training. Stain, hematoxylin and eosin (magnification, x20).
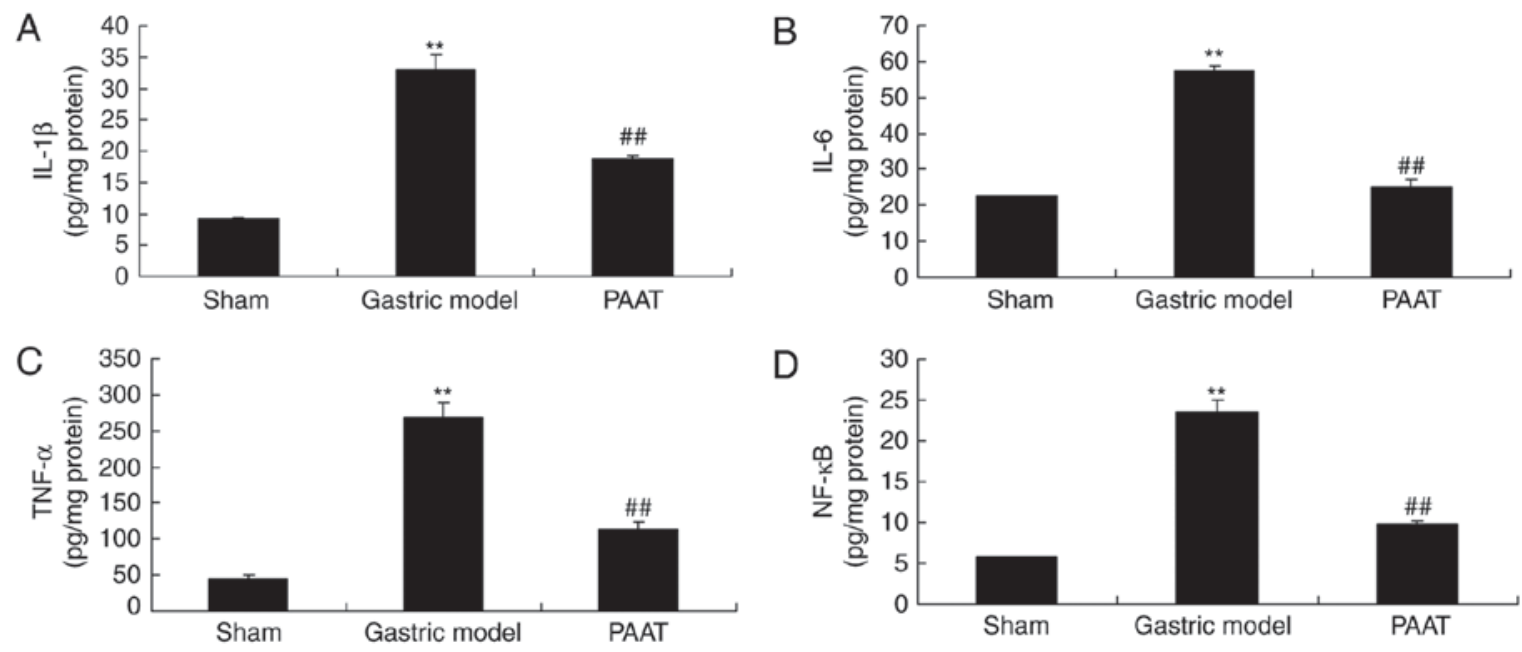

Figure 2. PAAT alleviates inflammatory factors in rats with GI-RI. PAAT reduced the serum levels of (A) IL-1 $\beta$, (B) IL-6, (C) TNF- $\alpha$ and (D) NF- $\kappa$ B in rats with GI-RI. ${ }^{* *} \mathrm{P}<0.01$ vs. the sham group; ${ }^{\# \#} \mathrm{P}<0.01$ vs. the gastric model group. PAAT, positive acceleration adaptive training; GI-RI, gastric ischemia-reperfusion injury; IL, interleukin; TNF- $\alpha$, tumor necrosis factor- $\alpha$; NF- $\kappa \mathrm{B}$, nuclear factor- $\kappa \mathrm{B}$.

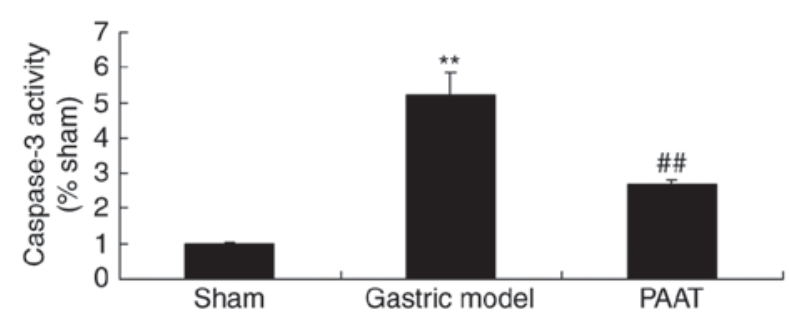

Figure 3. PAAT suppresses caspase-3 activity in rats with gastric ischemia-reperfusion injury. ${ }^{* *} \mathrm{P}<0.01$ vs. the sham group; ${ }^{\# \#} \mathrm{P}<0.01$ vs. the gastric model group. PAAT, positive acceleration adaptive training.

PAAT suppresses TNF- $\alpha$ and TNFR1 protein expression levels in rats with GI-RI. The protein expression levels of TNF- $\alpha$ and TNFR1 in rats with GI-RI were significantly higher than those of the sham group (Fig. 4). PAAT significantly suppressed TNF- $\alpha$ and TNFR1 protein expression levels in rats with GI-RI compared with the levels in the GI-RI model group (Fig. 4). These results indicated that PAAT significantly suppressed TNF- $\alpha$ and TNFR1 protein expression levels, thus reducing inflammation in GI-RI.

PAAT suppresses TRAIL, DR4 and DR5 protein expression in rats with GI-RI. As demonstrated in Fig. 5, TRAIL, DR4 and DR5 protein expression levels in rats with GI-RI were significantly induced compared with the levels in the sham group. Furthermore, PAAT significantly suppressed TRAIL, DR4 and DR5 protein expression levels in rats with GI-RI compared with the levels in the GI-RI model group (Fig. 5). These data demonstrated that PAAT reduced inflammation in GI-RI via the regulation of TRAIL, DR4 and DR5 expression.

PAAT suppresses COX-2 and PGE2 protein expression levels in rats with GI-RI. As demonstrated in Fig. 6, COX-2 and PGE2 protein expression levels in rats with GI-RI were higher than those of the sham group. Additionally, PAAT significantly suppressed COX-2 and PGE2 protein expression levels. (Fig. 6). The results demonstrated that PAAT suppresses COX-2 and PGE2 protein expression levels to inhibit inflammation in GI-RI.

\section{Discussion}

GI-RI is common in critically ill patients (10). Neuroendocrine system dysfunction occurs under the stress of injury, burns, shock, major surgery and critical diseases. This results in increased catecholamine production, vasoconstriction, reduced blood volume, decreased cardiac output and the release of pro-inflammatory factors (14). Therefore, the blood may flow 
A TNF- $\alpha$
TNFR1
GAPDH
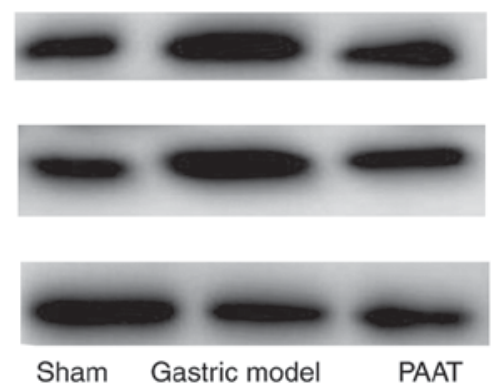
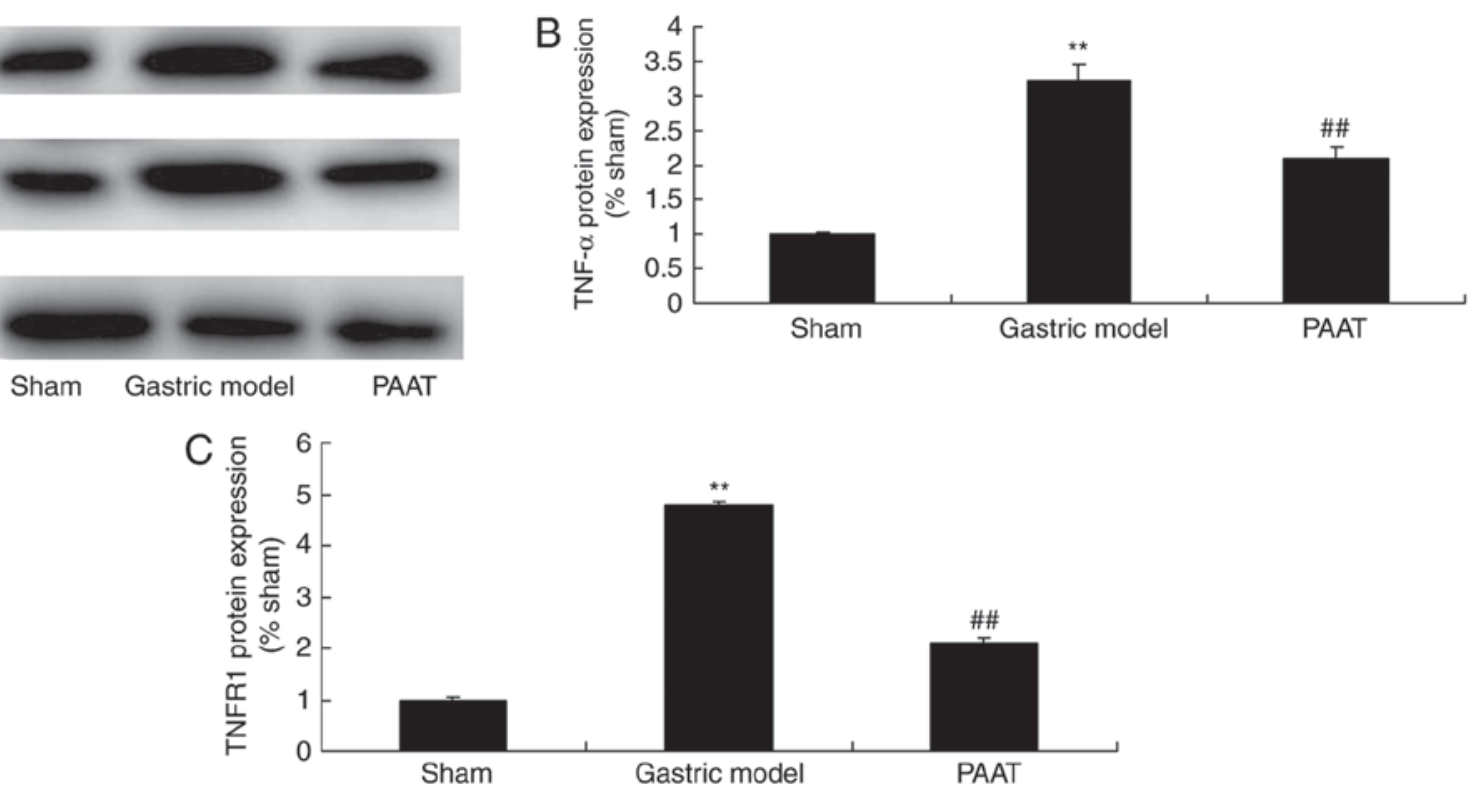

Figure 4. PAAT suppresses TNF- $\alpha$ and TNFR1 protein expression levels in rats with GI-RI. (A) Western blotting demonstrated that PAAT suppressed TNF- $\alpha$ and TNFR1 protein expression levels in rats with GI-RI. Western blotting results for (B) TNF- $\alpha$ and (C) TNFR1 were quantified. **P<0.01 vs. the sham group; ${ }^{\# \#} \mathrm{P}<0.01$ vs. the gastric model group. PAAT, positive acceleration adaptive training; GI-RI, gastric ischemia-reperfusion injury; TNF- $\alpha$, tumor necrosis factor- $\alpha$; TNFR1, tumor necrosis factor receptor 1 .
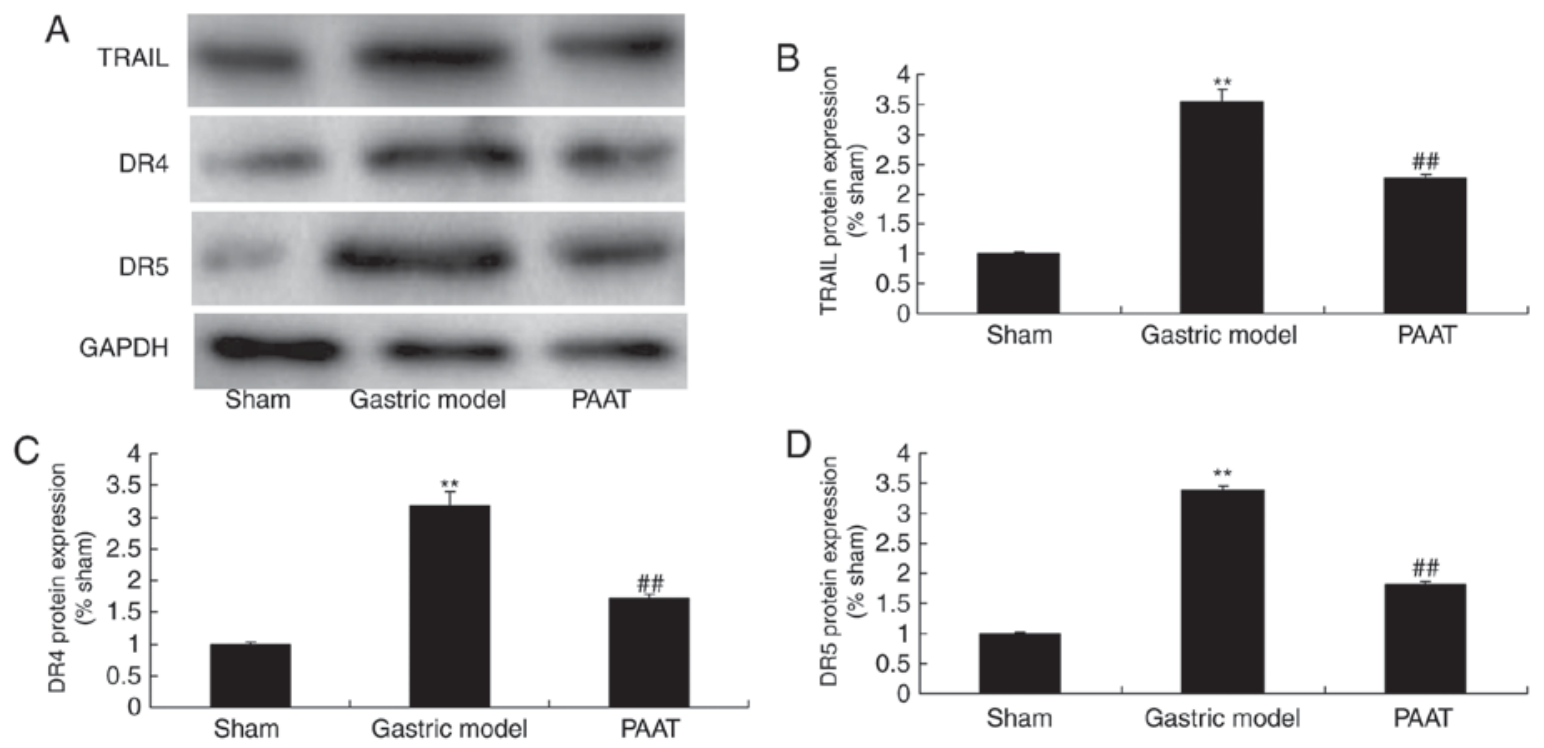

Figure 5. PAAT suppresses TRAIL, DR4 and DR5 protein expression in rats with GI-RI. (A) Western blotting demonstrated that PAAT suppressed TRAIL, DR4 and DR5 protein expression. (B) TRAIL, (C) DR4 and (D) DR5 protein expression levels were quantified. "* P $<0.01$ vs. the sham group; ${ }^{* \#} \mathrm{P}<0.01$ vs. the gastric model group. PAAT, positive acceleration adaptive training; GI-RI, gastric ischemia-reperfusion injury; TRAIL, tumor necrosis factor-related apoptosis inducing ligand; DR, death receptor.

into the heart, brain and muscles from the gastrointestinal tract and skin (7). In this manner, GI-RI may ensure a sufficient blood supply for vital organs. Gastrointestinal blood flow is reduced with disease progression. This leads to an insufficient oxygen supply and decreased bicarbonate secretion, thus damaging the gastric mucosa (12). Hydrogen ion reflux and pepsin erosion may further injure the mucosal epithelial layer in the presence of damage to the gastric mucosal layer (12). Slowing down the blood flow may affect the healing of gastric mucosa, accompanied with splanchnic perfusion deficiency and decreased gastrointestinal motion (1). Additionally, elimination of acids and other irritants in the stomach may be slowed down (6). Thus, the damaged gastric mucosa may be exposed to gastric acid for an increased time, which thereby increases the risk of ulcer formation (12). Nitric oxide (NO) synthase levels increase with reperfusion following prolonged ischemia, resulting in congestion and cell death, and promotion of the inflammatory response (2).

Numerous cytokines (including TNF- $\alpha$, NO, IL-1 and IL-8) are involved in GI-RI. Amongst them, TNF- $\alpha$ is the first and core endogenous inflammatory cytokine released from the inflammatory response. TNF- $\alpha$ is also the major cause 
A

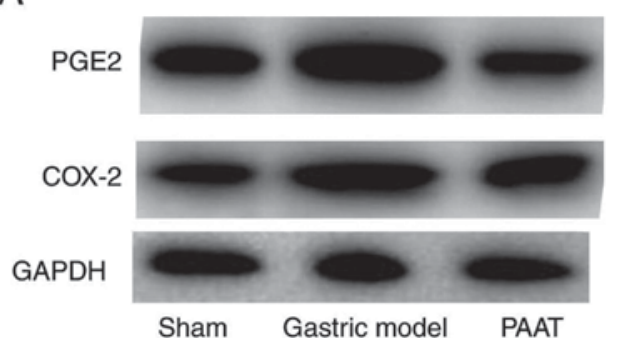

B

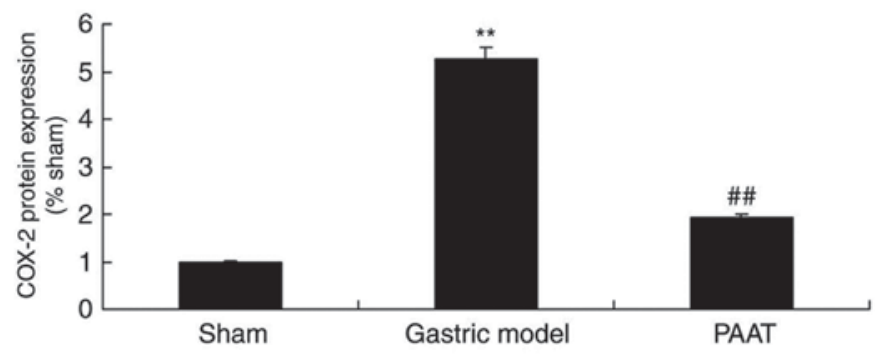

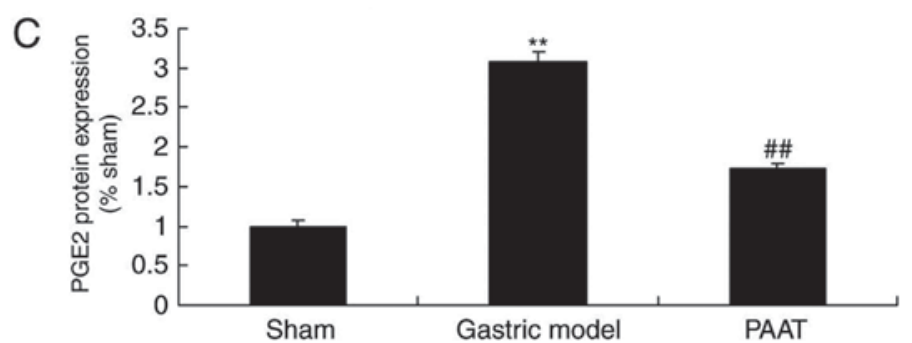

Figure 6. PAAT suppresses COX-2 and PGE2 protein expression levels in rats with GI-RI. (A) Western blotting demonstrated that PAAT suppressed protein expression levels of PGE2 and COX-2 in rats with GI-RI. (B) COX-2 and (C) PGE2 protein expression levels were quantified. ${ }^{* *}$ P $<0.01$ vs. the sham group; ${ }^{\# \#} \mathrm{P}<0.01$ vs. the gastric model group. PAAT, positive acceleration adaptive training; GI-RI, gastric ischemia-reperfusion injury; PGE2, prostaglandin E2; COX, cyclooxygenase.

of the inflammatory cascade reaction (6). Normal TNF- $\alpha$ levels serve an important role in the natural immune and host defense. However, excessive production and release of TNF- $\alpha$ may destroy the body's immunological equilibrium, which may cause a variety of pathological damages, along with other inflammatory factors (13). The main biological function of $\mathrm{TNF}-\alpha$ is to induce the inflammatory response by upregulating gene transcription (15). It is involved in numerous physiological and pathological responses via its receptor, TNFR1. Furthermore, it serves a vital role in inflammation, proliferation, differentiation and other pathophysiological processes (16). PAAT is known to reduce the inflammatory response (17). The present study indicated that PAAT significantly alleviated IL-1 $\beta$, IL- 6, TNF- $\alpha$ and NF- $\kappa$ B serum levels in rats with GI-RI.

The association between pathogenesis and the development of GI-RI apoptosis has not yet been fully elucidated. Nonetheless, the mechanism of apoptosis has always been a research hotspot and a source of debate. A previous study revealed that apoptosis has far-reaching significance and broad application prospects in the etiology and treatment of GI-RI science $(7,18)$. However, scholars have also identified another TNF family of TRAIL $(7,18)$.

TRAIL has been revealed in a previous study to lead to selective apoptosis of virus-infected tissues or cells and transformed cells (19). However, it may not affect the growth and differentiation of normal cells (19). FAS/FAS ligand- and TNFR1/TNF- $\alpha$-induced apoptosis regulates the DR signaling pathway (18). In addition, the corresponding DRs mainly include DR3, DR4 and DR5 (18). Amongst them, FAS and TNF- $\alpha$ belong to the TNF superfamily, which have been revealed to be closely associated with the incidence and development of GI-RI (20). Consistently, the present study demonstrated that PAAT significantly suppresses TNF- $\alpha$ and TNFR1 protein expression and reduces TRAIL, DR4 and DR5 protein expression levels in rats with GI-RI.
PG2 is a PG with complex biological activity, which is involved in GI-RI inflammation (19). The levels of PGE2, TNF- $\alpha$, IL-1 $\beta$ and leukotriene B4 have been demonstrated to increase in a rat model of Escherichia coli lipopolysaccharide-induced GI-RI (19). PGE2, which is a pro-inflammatory mediator, serves a regulatory role in lung injury (21). Furthermore, important cellular mechanisms of GI-RI include the recruitment and activation of neutrophils in the lungs (19). Additionally, the release of oxygen free radicals, protease, inflammatory mediators and macrophages, as well as capillary endothelial cell involvement, is also involved in GI-RI (22). The data in the present study indicated that PAAT significantly suppressed PGE2 protein expression levels in rats with GI-RI.

COX is a membrane-bound protein that mainly exists in microsomal membrane. The human COX-1 gene is $\sim 22.5 \mathrm{~kb}$ in length (7). Transcriptional regulation is the major regulation mode of COX-2 expression (7). Furthermore, the promoter and its activity are of great significance to the regulation of COX-2 transcription (23). The COX-2 promoter is $1 \mathrm{~kb}$ in length. However, the conserved sequence of COX-1 is not evident, with a promoter of $2.4 \mathrm{~kb}$ in length (21). Various activator molecules, including cytokines, serum or protein kinase $\mathrm{C}$, regulate the expression of two enzymes independently (21). Transcription factor sequences containing COX-2 promoters include GATA-1, NF- $\kappa$ B and IL-6 (21). The mechanism of COX-2 expression and regulation is complex, and varies between different cell types and divergent inducers (24). Macrophages are the main source of PGE2 induction and lead to the generation of the inflammatory response (6). COX-2 only exists in macrophages and fibroblasts in patients with gastric ulcers between necrosis and granuloma tissues (23). COX-2 is mainly responsible for the regulation of inflammation in GI-RI (23). The results of the present study revealed that PAAT significantly suppressed COX-2 protein expression levels and did not notably affect COX-1 protein expression in rats with GI-RI. The present study also demonstrated that 
PAAT is able to inhibit inflammation; however, its specific mechanism requires further investigation.

In summary, PAAT attenuated GI-RI and reduced inflammation via the downregulation of COX-2 and PGE2 expression. The results indicate that suppression of COX-2 and PGE2, but not COX-1, is involved in the alleviation of GI-RI by PAAT, which inhibits inflammation.

\section{Acknowledgements}

The present study was financially supported by Supported by the 12th Five-Year Major Scientific Research Program of General Logistics Department of PLA (grant no. AKJ11J004).

\section{Competing interests}

The authors declare that they have no competing interests.

\section{References}

1. Ward MG, Warner B, Unsworth N, Chuah SW, Brownclarke C, Shieh S, Parkes M, Sanderson JD, Arkir Z, Reynolds J, et al: Infliximab and adalimumab drug levels in Crohn's disease: Contrasting associations with disease activity and influencing factors. Aliment Pharmacol Ther 46: 150-161, 2017.

2. Bukowczan J, Warzecha Z, Ceranowicz P, Kusnierz-Cabala B and Tomaszewska R: Obestatin accelerates the recovery in the course of ischemia/reperfusion-induced acute pancreatitis in rats. PLoS One 10: e0134380, 2015.

3. Broekman MM, Coenen MJ, Wanten GJ, van Marrewijk CJ, Klungel OH, Verbeek AL, Hooymans PM, Guchelaar HJ, Scheffer H, Derijks LJ, et al: Risk factors for thiopurine-induced myelosuppression and infections in inflammatory bowel disease patients with a normal TPMT genotype. Aliment Pharmacol Ther 46: 953-963, 2017.

4. Del Carmen S, de Moreno de, LeBlanc A and LeBlanc JG: Development of a potential probiotic yoghurt using selected anti-inflammatory lactic acid bacteria for prevention of colitis and carcinogenesis in mice. J Appl Microbiol 121: 821-830, 2016.

5. Negroni A, Prete E, Vitali R, Cesi V, Aloi M, Civitelli F, Cucchiara S and Stronati L: Endoplasmic reticulum stress and unfolded protein response are involved in paediatric inflammatory bowel disease. Dig Liver Dis 46: 788-794, 2014.

6. Roblin X, Boschetti G, Williet N, Nancey S, Marotte H, Berger A, Phelip JM, Peyrin-Biroulet L, Colombel JF and Del Tedesco E: Azathioprine dose reduction in inflammatory bowel disease patients on combination therapy: An open-label, prospective and randomised clinical trial. Aliment Pharmacol Ther 46: 142-149, 2017.

7. Brzozowski T, Konturek PC, Konturek SJ, Sliwowski Z, Drozdowicz D, Stachura J, Pajdo R and Hahn EG: Role of prostaglandins generated by cyclooxygenase-1 and cyclooxygenase- 2 in healing of ischemia-reperfusion-induced gastric lesions. Eur J Pharmacol 385: 47-61, 1999.

8. Tlaskalová-Hogenová H, Stěpánková R, Kozáková H,Hudcovic T, Vannucci L, Tučková L, Rossmann P, Hrnčír T, Kverka M, Zákostelská Z, et al: The role of gut microbiota (commensal bacteria) and the mucosal barrier in the pathogenesis of inflammatory and autoimmune diseases and cancer: Contribution of germ-free and gnotobiotic animal models of human diseases. Cell Mol Immunol 8: 110-120, 2011.

9. Akahoshi T, Tanigawa T, Sarfeh IJ, Chiou SK, Hashizume M, Maehara Y and Jones MK: Selective cyclooxygenase (COX) inhibition causes damage to portal hypertensive gastric mucosa: Roles of nitric oxide and NF-kappaB. FASEB J 19: 1163-1165, 2005 .
10. Li J, Tang HL, Chen Y, Fan Q, Shao YT, Jia M, Wang JC and Yang CM: Malondialdehyde and SOD-induced changes of gastric tissues in acute gastric mucosal injury under positive acceleration. Genet Mol Res 14: 4361-4368, 2015.

11. Sarosiek I, Bashashati M, Alvarez A, Hall M, Shankar N, Gomez Y, McCallum RW and Sarosiek J: Lubiprostone accelerates intestinal transit and alleviates small intestinal bacterial overgrowth in patients with chronic constipation. Am J Med Sci 352: 231-238, 2016.

12. Yang Y, Sandhu HK, Zhi F, Hua F, Wu M and Xia Y: Effects of hypoxia and ischemia on microRNAs in the brain. Curr Med Chem 22: 1292-1301, 2015.

13. Livak KJ and Schmittgen TD: Analysis of relative gene expression data using real-time quantitative PCR and the 2(-Delta Delta C(T)) method. Methods 25: 402-408, 2001.

14. Gezginci-Oktayoglu S, Orhan N and Bolkent S: Prostaglandin-E1 has a protective effect on renal ischemia/reperfusion-induced oxidative stress and inflammation mediated gastric damage in rats. Int Immunopharmacol 36: 142-150, 2016.

15. Chen T, Kim CY, Kaur A, Lamothe L, Shaikh M, Keshavarzian A and Hamaker BR: Dietary fibre-based SCFA mixtures promote both protection and repair of intestinal epithelial barrier function in a Caco-2 cell model. Food Funct 8: 1166-1173, 2017.

16. Bibi S, Kang Y, Du M and Zhu MJ: Dietary red raspberries attenuate dextran sulfate sodium-induced acute colitis. J Nutr Biochem 51: 40-46, 2018.

17. Wang XZ, Jiang WD, Feng L, Wu P, Liu Y, Zeng YY, Jiang J, Kuang SY, Tang L, Tang WN and Zhou XQ: Low or excess levels of dietary cholesterol impaired immunity and aggravated inflammation response in young grass carp (Ctenopharyngodon idella). Fish Shellfish Immunol 78: 202-221, 2018.

18. Devkota $\mathrm{S}$ and Chang EB: Interactions between diet, Bile acid metabolism, gut microbiota, and inflammatory bowel diseases. Dig Dis 33: 351-356, 2015.

19. Lin CC, Lin WN, Cho RL, Wang CY, Hsiao LD and Yang CM: TNF- $\alpha$-induced cPLA $_{2}$ expression via NADPH oxidase/reactive oxygen species-dependent NF- $\kappa \mathrm{B}$ cascade on human pulmonary alveolar epithelial cells. Front Pharmacol 7: 447, 2016

20. Zhang F, Xu C, Ning L, Hu F, Shan G, Chen H, Yang M, Chen W, $\mathrm{Yu}$ J and $\mathrm{Xu}$ G: Exploration of serum proteomic profiling and diagnostic model that differentiate Crohn's disease and intestinal tuberculosis. PLos One 11: e0167109, 2016.

21. Cheng L, Jin H, Qiang Y, Wu S, Yan C, Han M, Xiao T, Yan N, An H, Zhou X, et al: High fat diet exacerbates dextran sulfate sodium induced colitis through disturbing mucosal dendritic cell homeostasis. Int Immunopharmacol 40: 1-10, 2016.

22. Battison AL, Després BM and Greenwood SJ: Ulcerative enteritis in Homarus americanus: Case report and molecular characterization of intestinal aerobic bacteria of apparently healthy lobsters in live storage. J Invertebr Pathol 99: 129-135, 2008.

23. Ichimasa K, Kudo SE, Mori Y, Misawa M, Matsudaira S, Kouyama Y, Baba T, Hidaka E, Wakamura K, Hayashi T, et al: Artificial intelligence may help in predicting the need for additional surgery after endoscopic resection of T1 colorectal cancer. Endoscopy 50: C2, 2018.

24. Watson H, Cockbain AJ, Spencer J, Race A, Volpato M, Loadman PM, Toogood GJ and Hull MA: Measurement of red blood cell eicosapentaenoic acid (EPA) levels in a randomised trial of EPA in patients with colorectal cancer liver metastases. Prostaglandins Leukot Essent Fatty Acids 115: 60-66, 2016.

(i) $\Theta$ This work is licensed under a Creative Commons Attribution-NonCommercial-NoDerivatives 4.0 International (CC BY-NC-ND 4.0) License. 